\title{
Método rápido de determinación del sexo en el erizo comestible Loxechinus albus (Molina, 1782) y su aplicación en estudios biológico-pesqueros
}

\author{
Sergio Palma G. y Patricio Arana E. \\ Escuela de Ciencias del Mar \\ Universidad Católica de Valparaíso \\ Casilla 1020, Valparaíso, Chile
}

\begin{abstract}
RESUMEN. La identificación del sexo en el erizo comestible Loxechinus albus (Molina, 1782), es difícil de realizar si no se realiza un análisis de laboratorio, lo que motiva que en la mayoría de las investigaciones efectuadas sobre este recurso se consideren ambos sexos en conjunto. En el presente artículo se describe una metodología simple para establecer el sexo en el erizo, lo que permite obtener parámetros biológico-pesqueros en forma separada para machos y hembras. A través de los procedimientos que se describen, es posible separar las distribuciones de frecuencias de tallas obtenidas en los muestreos, sin diferenciar el sexo, en las correspondientes a machos y hembras. Este procedimiento es especialmente útil cuando los sexos presentan diferencias de crecimiento o mortalidad.

Palabras claves: determinación sexo, erizo comestible, Loxechinus albus, Chile

\section{Simple methodology to identify sex of sea urchin Loxechinus albus (Molina, 1782), and its application to fishery-biological studies.}

\begin{abstract}
Sex identification of the edible sea urchin Loxechinus albus (Molina, 1782), is difficult if not done in a laboratory, therefore most of the studies on this resource does not consider sexes separately. This paper describes a simple methodology to identify sex of the sea urchin, enabling the estimation of fishery-biological parameters separately for males and females. Through the described procedures, it is possible to separate by sex the length frequency distributions from samples taken without differentiating males from females. This procedure becomes specially useful when sexes show differences in growth or mortality.
\end{abstract}

Key words: sex determination, sea urchin, Loxechinus albus, Chile

\section{INTRODUCCION}

El erizo blanco o comestible Loxechinus albus (Molina, 1782), es un equinodermo que se distribuye a lo largo de la costa suroriental del oceáno Pacífico, desde el Perú (isla Lobos de Afuera, 6 $53^{\prime} 50 »$ S), hasta el extremo austral de Chile (Cabo de Hornos, $\left.55^{\circ} 52^{\prime \prime S}\right)$ y Tierra del Fuego en el sector argentino (Guisado y Castilla, 1987). Esta especie habita en fondos duros y se distribuye desde la zona litoral hasta los $340 \mathrm{~m}$ de profundidad (Larraín, 1975); sin embargo su extracción se concentra entre los 0 y $40 \mathrm{~m}$ de profundidad.

Esta es la única especie de erizo que se explota comercialmente en el país. A pesar que se captura a lo largo de toda la costa, en los últimos años el es- fuerzo pesquero se ha concentrado principalmente en la región sur- austral de Chile $\left(42^{\circ}-57^{\circ} \mathrm{S}\right)$, lo que ha motivado un notorio incremento en los desembarques de esa zona. Esta situación ha originado preocupación, a fin de asegurar la sustentabilidad del recurso en aquellas zonas en que se está extrayendo en forma intensiva.

En la actualidad, las medidas de manejo implementadas para la protección de esta especie, se orientan a la fijación de una talla mínima de extracción de $7 \mathrm{~cm}$ de diámetro máximo, sin incluir las púas (Decreto $\mathrm{N}^{\circ} 291$, 30/sep/91, del Ministerio de Economía, Fomento y Reconstrucción, D.O. $\mathrm{N}^{\circ} 32.891,10 /$ oct/91), y al establecimiento de períodos de veda. En la zona norte y centro-sur, se prohíbe su extracción entre el 15 de octubre de cada 
año, y el 15 de enero del año siguiente (Decreto N²91, 30/sep/91, del Ministerio de Economía, Fomento y Reconstrucción, D.O. N³2.891, 10/oct/91), mientras que en la región sur-austral, la veda extractiva se extiende desde el 15 de agosto al 30 de noviembre de cada año (Decreto $\mathrm{N}^{\circ} 166$ exento, 23/ sep/94, del Ministerio de Economía, Fomento y Reconstrucción, D.O. N³4.976, 27/sep/94),

El erizo se caracteriza por su caparazón hemisférico de color verde, con tonalidades rojizas o moradas en ambulacros e interambulacros. Es una especie gonocórica, es decir, tiene los sexos separados, aunque no presenta dimorfismo sexual y tiene actividad gametogénica a partir de los 20 a $25 \mathrm{~mm}$ de diámetro máximo. No obstante, a nivel poblacional se estima que el tamaño de primera madurez en las hembras se produce a los $45 \mathrm{~mm}$, mientras que los machos la alcanzan a los $55 \mathrm{~mm}$ (Bay-Schmith et al., 1981; SUBPESCA, 1994).

La época de reproducción se extiende entre noviembre y diciembre en la zona norte de Chile, mientras que en la zona central y sur ocurre entre julio y diciembre. Dada la ausencia de dimorfismo sexual la separación de sexos es muy difícil de realizar sin un examen histológico. Más aún, las gónadas de machos y hembras tienen la misma estructura externa y coloración; aunque ésta última puede variar del amarillo al café oscuro. Según Bückle (1973), la identificación sexual se puede lograr en forma aproximada sólo en el período de máxima actividad reproductiva, cuando de las gónadas de las hembras fluye un líquido de color anaranjado que contiene los óvulos; mientras que de los machos fluye un líquido blanco constituido por los espermios.

A la actualidad, la separación de machos y hembras ha sido realizada mediante el estudio histológico de sus gónadas (Gutiérrez y Otsu, 1975; BaySchmith et al.,1981). Sin embargo, esta técnica es demasiado lenta y no permite el análisis rápido de un número importante de ejemplares, como es el requerimiento de diversas metodologías biológicopesqueras destinadas a analizar muestras de capturas comerciales.

En el presente trabajo se describe una nueva metodolgía que permite determinar el sexo en forma rápida y a bajo costo, y además estimar la proporción sexual en muestras de gran tamaño. Así también, se entrega el procedimiento para separar distribuciones globales de frecuencias de tallas realizadas sin diferenciación sexual, en distribuciones separadas para machos y hembras, para su subsecuente utilización en estudios biológicos y pesqueros.

\section{MATERIALES Y METODOS}

\section{Material examinado}

Los erizos analizados se obtuvieron de capturas comerciales realizadas por pescadores artesanales en la región de Magallanes, entre junio y noviembre de 1995, período señalado como de reproducción en esta especie (Bay-Schmith et al., 1981). A cada ejemplar se le midió el diámetro máximo con $1 \mathrm{~mm}$ de precisión. Las frecuencias de tallas fueron agrupadas en intervalos de $5 \mathrm{~mm}$, sin diferenciación de sexo (Arana et al., 1996).

\section{Determinación del sexo y proporción sexual a la talla}

Para determinar el sexo de los individuos, se agruparon los ejemplares comerciales en 10 rangos de longitud, entre los 65 y 115 mm de diámetro máximo. En cada rango de $5 \mathrm{~mm}$ de diámetro se midió y separó un total aproximado de 60 individuos. A cada uno de los ejemplares se le extrajo una de las cinco gónadas o "lenguas", la que fue fijada en un frasco con formalina al $5 \%$ para su conservación y posterior análisis en laboratorio.

La separación de las gónadas correspondientes a machos y hembras, se efectuó por observación macroscópica de frotis gonadales. Para lo cual, a cada gónada se le extrajo una pequeña fracción y se efectuó una preparación en un portaobjeto. Luego, los frotis fueron teñidos utilizando azul de metileno, azul de toluidina y hematoxilina-eosina. La observación macroscópica de las gónadas, como de los frotis gonadales fue efectuada con un microscopio estereoscópico Wild M8, con máquina fotográfica Wild MPS12.

Paralelamente, se efectuaron preparaciones histológicas para determinar el sexo de las gónadas. De esta manera, se procedió a validar las determinaciones efectuadas mediante el análisis macroscópico antes descrito.

Una vez determinado el sexo de cada gónada, se contabilizó el número total de machos y hembras contenidos en la muestra y con estos valores, se pro- 
cedió a determinar la proporción sexual en cada intervalos de $5 \mathrm{~mm}$ de longitud. La varianza en cada rango se determinó como $\operatorname{var}(\hat{\mathrm{p}})=\mathrm{p}(1-\mathrm{p}) / \mathrm{n}$.

\section{Reestructuración de las frecuencias de tallas por sexos}

Una vez conocida la proporción sexual a la talla, se utilizaron dichos valores para separar las distribuciones de frecuencias de tallas obtenidas en los muestreos en terreno, en las correspondientes a machos y hembras. Para esto, se multiplicó la proporción correspondiente a cada sexo por el número total de ejemplares en cada intervalo de longitud.

Para el manejo de los registros de las distribuciones de frecuencias de tallas y procesos estadísticos se utilizaron planillas de cálculo.

\section{RESULTADOS Y DISCUSION}

En una amplia gama de recursos marinos se ha demostrado que existe una amplia variación en la proporción sexual a la talla, siendo el trabajo de Wenner (1972), uno de los precursores en esta línea de investigación. Estas diferencias en los porcentajes de machos y hembras, en cada rango de longitud, pueden ser atribuidas a diversos factores como el comportamiento o distribución, diferencia de los individuos o bien a diferencias en el reclutamiento, crecimiento o mortalidad entre los sexos. De allí que la determinación del sexo en los análisis biológicopesqueros cobran especial importancia, especialmente para el cálculo de las constantes de crecimiento, los que van a incidir en forma decisiva en la determinación de las mortalidades y otros parámetros utilizados en evaluación de poblaciones.

En el erizo, la coloración de las gónadas muestra una variada gama de tonalidades, que va desde el amarillo, amarillo-naranja, naranja, café claro al café oscuro. Estas tonalidades están relacionadas con el estado de madurez de los individuos (Gutiérrez y Otsu, 1975), pero no permiten diferenciar las células sexuales. Dado que la morfología externa de las gónadas es muy similar en ambos sexos, la observación macroscópica de las gónadas, fracciones de éstas o frotis gonadales no permiten distinguir machos de hembras.

En cambio, el empleo de tinciones permitió observar diferencias importantes entre los sexos, tanto en la consistencia del tejido, como en la colora- ción de los frotis gonadales. En este sentido, los mejores resultados se obtuvieron en los frotis teñidos con azul de metileno.

En las gónadas correspondientes a los machos se observó un tejido homogéneo, de consistencia cremosa y estructuras poco definidas, de intenso color azul-verdoso oscuro (Figs. 1a y 1b). Mientras que en las gónadas de las hembras se detectó un tejido granuloso, de consistencia heterogénea, con formación de numerosos aglomerados causados por la presencia de ovocitos, presentando una coloración violeta, pero que en las fotografías se observó de color café (Figs. 2a y 2b). En este mismo sexo, la observación macroscópica mostró, nítidamente, la presencia de ovocitos repartidos en toda la gónada, de formas y tamaños muy variados. Estas observaciones fueron comprobadas con análisis histológicos de cortes gonadales.

Para ejemplificar el procedimiento utililizado para la separación de machos y hembras en las capturas comerciales, se empleó la información correspondiente al muestreo efectuado en julio de 1995, mes en que se midieron 25.709 ejemplares sin distinción de sexos. Una vez medidos los ejemplares, se obtuvo una submuestra de 576 ejemplares entre los $65 \mathrm{~mm}$ y los $115 \mathrm{~mm}$, separados en diez clases de talla. Mediante el examen de las gónadas se estableció la proporción sexual en los distintos rangos de tallas, resultando porcentajes de machos comprendidos entre $31,2 \%$ y 76,7\% (Tabla 1 y Fig. 3). Dichos valores se utilizaron para determinar el número de machos y hembras en cada rango y de allí disponer de la distribución de frecuencia de tallas de cada sexo (Fig. 4).

El procedimiento descrito permite un trabajo más rápido en terreno, a la vez que permite posteriormente, disponer de las distribuciones de frecuencia de tallas de cada uno de los sexos. Al contar con estos registros es factible trabajar en forma separada para determinar, por ejemplo, la proporción sexual de la muestra global, la talla media o la fracción de ejemplares que se encuentra sobre, bajo o entre determinadas tallas (Tabla 2).

A pesar que el análisis histológico constituye una forma tradicional para efectuar la identificación sexual, las ventajas de la metodología descrita parecen evidentes, particularmente cuando los ejemplares se encuentran en proceso de maduración. Al igual que en el erizo, este procedimiento se puede aplicar a otros recursos que no presentan dimorfis- 

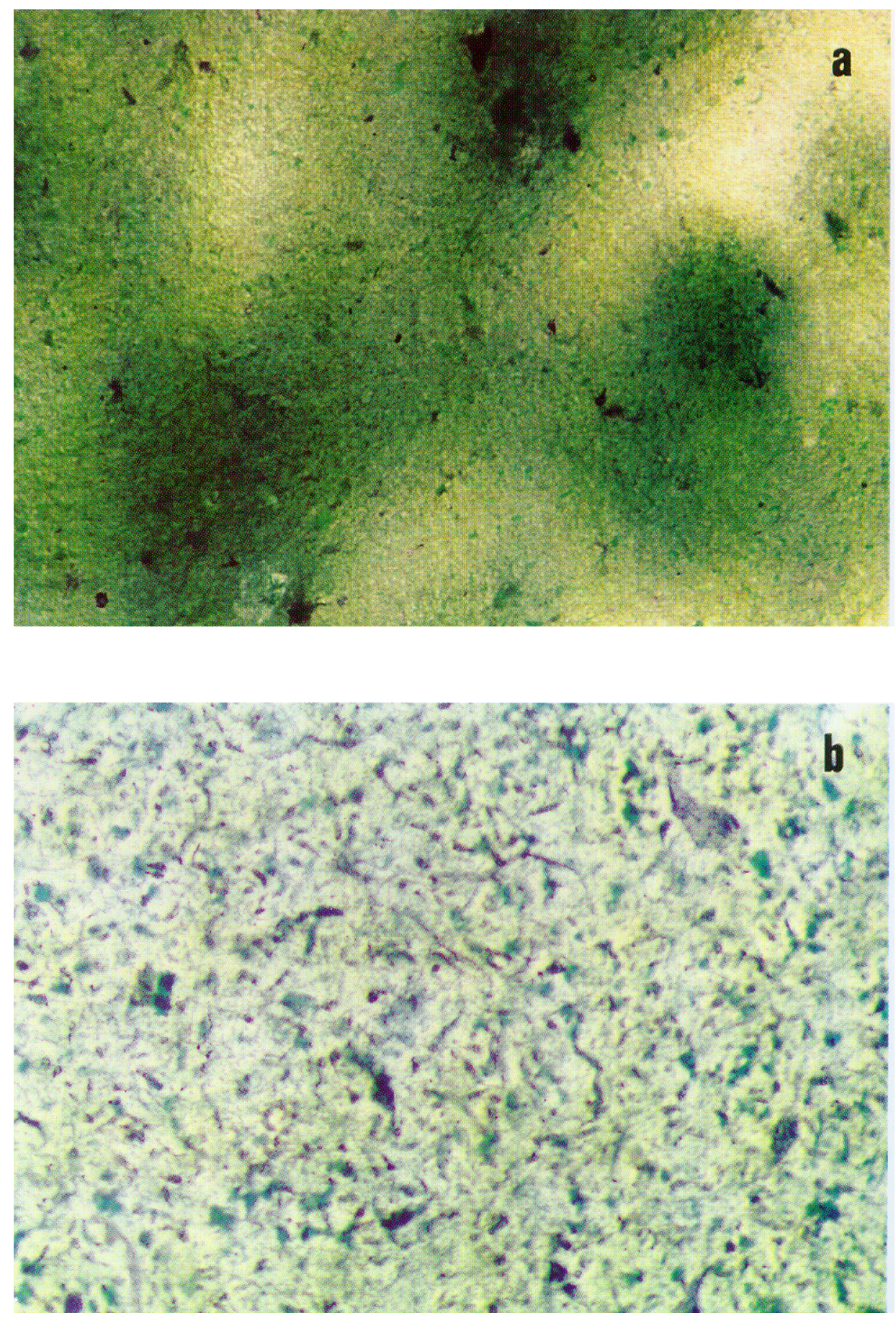

Figura 1. Observación macroscópica de gónadas machos de erizo. a) 60x; b) 400x.

Figure 1. Macroscopic observation of sea urchin male gonads. a) 60x; b) 400x. 

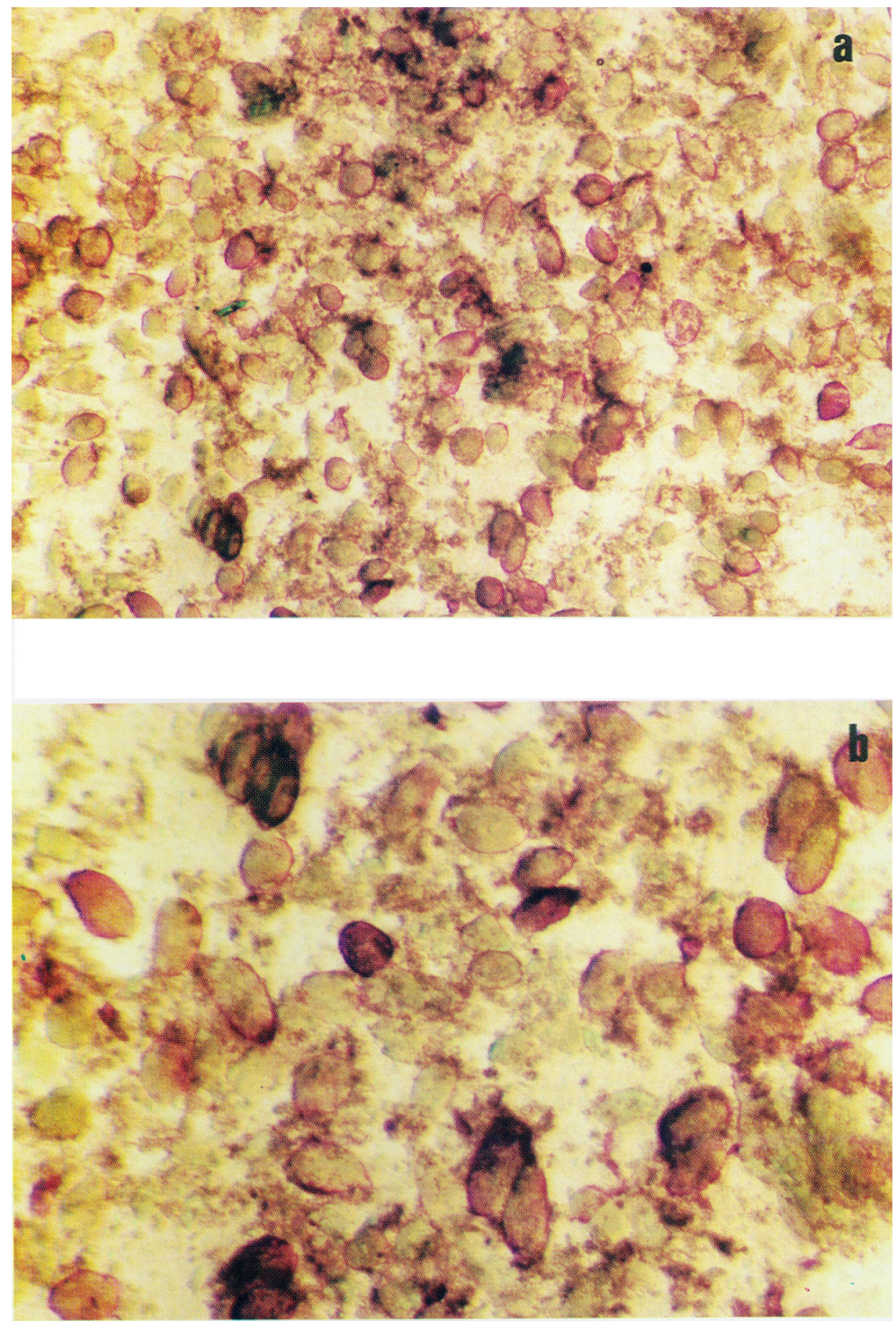

Figura 2. Observación macroscópica de gónadas hembras de erizo. a) Ovocitos 250x; b) Ovocitos 500x. Figure 2. Macroscopic observation of sea urchin female gonads. a) Oocytes 250x; b) Oocytes 500x. 
Tabla 1. Distribución de frecuencias de tallas sin sexar, proporción de macho por rango de longitud y distribución de frecuencias por sexo.

Table 1. Size frequency distribution of individuals without sex identification, males proportion by length range and frequency distribution for each sex.

\begin{tabular}{|c|c|c|c|c|c|c|c|c|}
\hline \multirow{2}{*}{$\begin{array}{l}\text { Rango diámetro } \\
\text { máximo }(\mathrm{mm})\end{array}$} & \multirow{2}{*}{$\begin{array}{c}\text { Marca de } \\
\text { clase }(\mathrm{mm})\end{array}$} & \multirow{2}{*}{$\begin{array}{c}\text { Gónadas } \\
\text { examinadas }\end{array}$} & \multicolumn{2}{|c|}{ Identificación del sexo } & \multirow{2}{*}{$\begin{array}{c}\text { Proporción de } \\
\text { machos (p) }\end{array}$} & \multirow{2}{*}{$\begin{array}{c}\text { N en muestra } \\
\text { sin sexar }\end{array}$} & \multicolumn{2}{|c|}{ Frecuencia calculada } \\
\hline & & & machos & hembras & & & machos & hembras \\
\hline $65,0-69,9$ & 67,5 & 60 & 36 & 24 & 0,60 & 1767 & 1060 & $\dot{707}$ \\
\hline $70,0-74,9$ & 72,5 & 60 & 45 & 15 & 0,75 & 5000 & 3750 & 1250 \\
\hline $75,0-79,9$ & 77,5 & 60 & 19 & 41 & 0,32 & 5852 & 1853 & 3999 \\
\hline $80,0-84,9$ & 82,5 & 67 & 24 & 43 & 0,36 & 5671 & 2030 & 3641 \\
\hline $85,0-89,9$ & 87,5 & 60 & 36 & 24 & 0,60 & 4241 & 2545 & 1696 \\
\hline $90,0-94,9$ & 92,5 & 65 & 30 & 35 & 0,46 & 2090 & 965 & 1125 \\
\hline $95,0-99,9$ & 97,5 & 66 & 26 & 40 & 0,39 & 892 & 351 & 541 \\
\hline $100,0-104,9$ & 102,5 & 61 & 19 & 42 & 0,31 & 125 & 39 & 86 \\
\hline $105,0-109,9$ & 107,5 & 60 & 46 & 14 & 0,77 & 26 & 20 & 6 \\
\hline $110,0-114,9$ & 112,5 & 17 & 11 & 6 & 0,65 & 45 & 29 & 16 \\
\hline Total & & 576 & & & & 25709 & 12642 & 13067 \\
\hline
\end{tabular}

Tabla 2. Resultados estadísticos de muestras sin sexar y separadas por sexo.

Table 2. Statistical results for samples with and without sex identification.

\begin{tabular}{|l|c|c|c|}
\hline & \multirow{2}{*}{$\begin{array}{c}\text { Muestra sin sexar } \\
\text { (machos+hembras) }\end{array}$} & \multicolumn{2}{|c|}{ Muestra separada por sexo } \\
\cline { 3 - 4 } & 25.715 & 13.071 & 12.644 \\
Número de ejemplares (n) & - & 49,2 & 50,8 \\
Proporción sexual & 80,72 & 79,90 & 81,51 \\
Talla media (diámetro máximo, mm) & 77,5 & 72,5 & 77,5 \\
Moda (mm) & 6,9 & 8,4 & 5,4 \\
Porcentaje de ejemplares bajo la talla & &
\end{tabular}

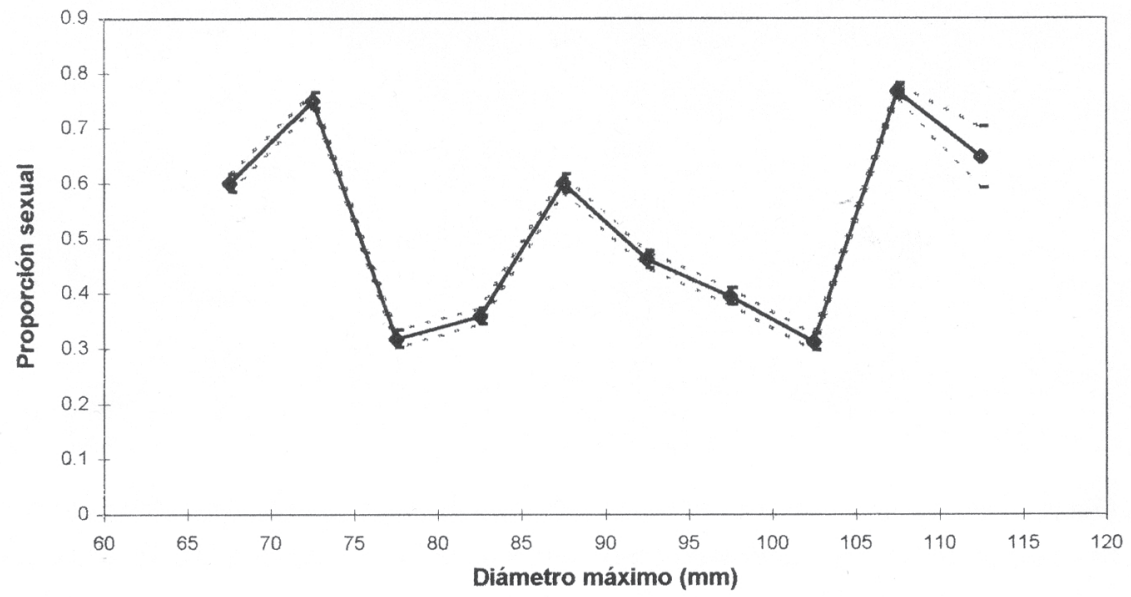

Figura 3. Proporción sexual a la talla en erizos extraídos en la región de Magallanes (julio de 1995), determinada a través del análisis macroscópico de frotis gonadales teñidos. Se indica el intervalo de confianza al $95 \%$.

Figure 3. Sexes proportion at length of sea urchin caught in the Magellan region (July 1995), determined by macroscopic analysis of gonadal dyed smears (95\% confidence interval is shown). 

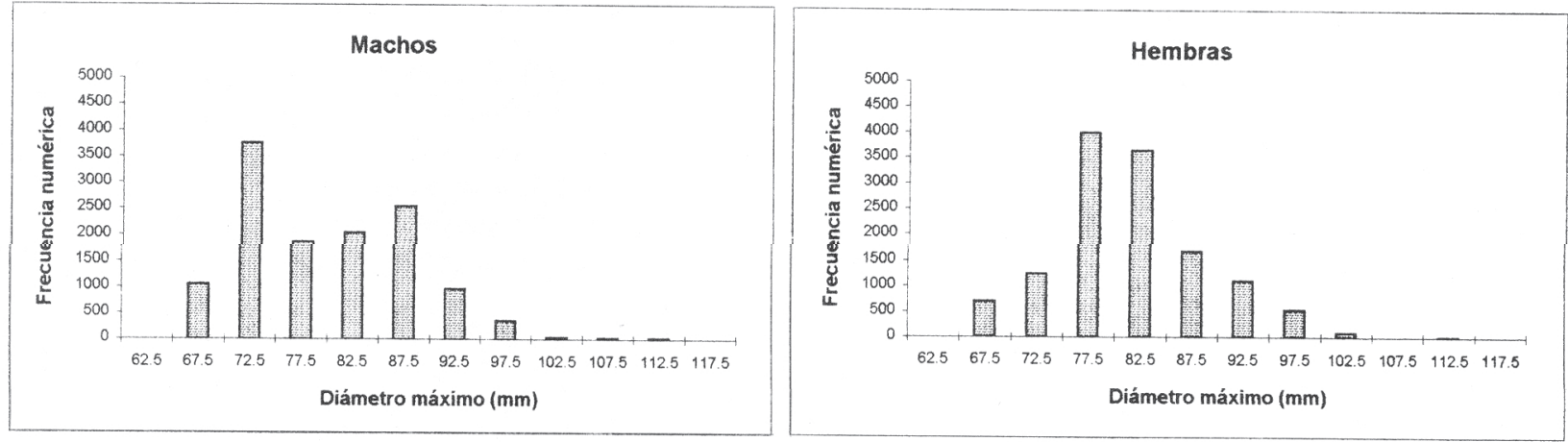

Figura 4. Distribución de frecuencias de tallas sin sexar determinadas a través de mediciones efectuadas en terreno y las correspondientes a machos y hembras, separadas mediante el procedimiento descrito en el texto.

Figure 4. Size frequency distribution of field measurements for individuals without sex identification and for males and females separated by the present methodology.

mo sexual. Además, su aplicación parece fundamental cuando se sospecha que existen diferencias de crecimiento o de mortalidad entre ambos sexos.

De acuerdo a las necesidades de cada investigación, las muestras pueden obtenerse en el rango de talla que se desee, para lo cual se debe tomar una muestra en cada intervalo utilizado. El tamaño de muestra en cada caso queda definido por la expresión $\mathrm{n}=\left[\mathrm{z}_{(1-\alpha / 2)}^{2} * \mathrm{p}(1-\mathrm{p})\right] / \varepsilon^{2}$.

Cabe destacar que en la mayoría de las investigaciones realizadas sobre esta especie, se han con- siderado conjuntamente machos y hembras, existiendo sólo algunos estudios específicos en que se han sexado los individuos. Así por ejemplo, en todos los trabajos realizados en el país acerca del crecimiento del erizo, se han considerado muestras sin sexar, entregándose parámetros únicos para este recurso (Bückle et al., 1977; Zegers et al., 1983; Zabala, 1987; González et al., 1990; Araya et al., 1991; Gebauer, 1992; Gebauer y Moreno, 1995). No obstante, utilizando la metodología aquí descrita se ha determinado que existen diferencias en el crecimiento y mortalidad entre machos y hembras (Gálvez, 1996). 
Por el motivo antes indicado, es indispensable considerar la identificación del sexo para determinar parámetros poblacionales y realizar los análisis biológico-pesqueros en este recurso.

\section{AGRADECIMIENTOS}

Los autores dejan expresa constancia del apoyo logístico y financiero otorgado por la empresa Comercial y Pesquera Hanamar Ltda. y en especial a su dueño, Sr. Hitochi Hanaoka, como también al Sr. Fernando Concha E., por su constante incentivo por ampliar el conocimiento del recurso erizo de la región de Magallanes. Igualmente, los autores agradecen a la Srta. Lorena Linacre R. y Sr. Sergio Rosales G., por su colaboración en la preparación y análisis de las muestras.

\section{REFERENCIAS}

Araya, M., M. Medina y M. Gallardo. 1992. Crecimiento en el medio natural del erizo comestible (Loxechinus albus) de la zona de Iquique. Informe Final, Depto. de Ciencias del Mar, Univ. Arturo Prat, 34 pp.

Arana, P., S. Palma, E. Bay-Schmith, M.A. Monardes y M. Gálvez. 1996. Aspectos biológicos y pesqueros del erizo (Loxechinus albus) en la región de Magallanes. Estud. Doc., Univ. Católica Valparaíso, 23/96: 83-140 pp.

Bay-Schmith, E., C. Werlinger y J. Silva. 1981. Ciclo anual de reproducción del recurso Loxechinus albus entre la X y XIIa Región. Informe Final Proyecto de Investigación, Subsecretaría de PescaUniversidad de Concepción, 68 pp.

Bückle, L. 1973. El erizo comestible, (Loxechinus albus (Mol.)) (Echinoidea: Echinoidea). Noticiero Mensual, Mus. Nac. Hist. Nat., Chile, 17(205): 811.

Bückle, F., C. Guisado, C. Serrano, L. Cordova, L. Peña y E. Vásquez. 1977. Estudio del crecimiento en cautiverio del erizo Loxechinus albus (Molina) en las costas de Valparaíso y Chiloé. Anales del Centro de Ciencias del Mar y Limnología, Universidad Nacional Autónoma de México, 4(1): 141152.

Recibido el 30 de noviembre de 1996. Aceptado el 15 de marzo de 1997.
Gálvez, M. 1996. Evaluación indirecta del stock de erizo comestible (Loxechinus albus) al sur del estrecho de Magallanes (Chile), durante la temporada 1995. Estud. Doc., Univ. Católica Valparaíso, 23/96: 254-286 pp.

Gebauer, P. 1992. Validación experimental de los anillos de crecimiento de Loxechinus albus (Molina, 1782) (Echinodermata: Echinoidea) en la reserva marina de Mehuin, Chile. Tesis, Esc. Bilogía Marina, Univ. Austral de Chile, 66 pp.

Gebauer, P. and C.A. Moreno.1995. Experimental validation of the growth rings of Loxechinus albus (Molina, 1782) in southern Chile (Echinodermata: Echinoidea). Fish. Res., 21: 423-435.

Gónzalez, M., M. Pérez; D. López y J. Uribe. 1990. Crecimiento del erizo Loxechinus albus (Molina), en condiciones artificiales. Biota, Osorno, Chile, 6: 35-44.

Guisado, C. y J.C. Castilla. 1987. Historia de vida, reproducción y avances en el cultivo del erizo comestible chileno Loxechinus albus (Molina, 1782) (Echinoidea: Echinidae). In: "Manejo y Desarrollo Pesquero", P. Arana (Ed.), Esc. Ciencias del Mar, UCV, Valparaíso, 59-68.

Gutiérrez, J. y I. Otsu. 1975. Periodicidad en las variaciones biométricas de Loxechinus albus Molina. Rev. Biol. Marina, Valparaíso, 15(2): 179-199.

Larraín, A. 1975. Los equinoideos regulares fósiles y recientes de Chile. Gayana, zool., 35:1-189 pp.

Subsecretaría de Pesca (SUBPESCA). 1994. Medidas de administración para la pesquería del recurso erizo (Loxechinus albus) en la XII Región . Informe Técnico, Subsecretaría de Pesca, 30 pp.

Wennner, A. 1972. Sex ratio as function of size in marine crustacea. Amer. Natur., 106: 321-351.

Zabala, A. 1987. Determinación de una metodología para establecer edad en el erizo comestible Loxechinus albus (Molina, 1782). Tesis, Univ. Católica, Sede Talcahuano, 54 pp.

Zegers, J., M. Oliva, C. hidalgo y L. Rodríguez. 1983. Crecimiento de Loxechinus albus (Molina, 1782) (Echinodermata: Echinoidea) en sistema de jaulas suspendidas a media agua. Mem. Asoc. Latinoam. Acuicult., 5(2): 369-378. 